\title{
The association of HDL-apoCIII with coronary heart disease and the effect of statin treatment on it
}

\author{
Xiaowei Xiong ${ }^{1}$, Hong Liu', Lu Hua', Hui Zhao' ${ }^{1}$ Dongxue Wang ${ }^{2}$ and Yishi Li ${ }^{\text {* }}$
}

\begin{abstract}
Background: Apolipoprotein CIII (apoCIII) is considered to impair the anti-atherogenic effect of high density lipoprotein $(\mathrm{HDL})$ in coronary heart disease (CHD) patients, and apoCIII content in $\mathrm{HDL}$ (HDL-apoCIII) predicts CHD more accurately. However, the relationship between HDL-apoCIII and CHD, and the effect of statin treatment on HDL-apoCIII are still unclear. The aims of the study are to establish the association of HDL-apoCIII with CHD, and investigate the effect of statin treatment on HDL-apoCIII in CHD patients.

Methods: We conducted a hospital-based observational study. Totally 80 non-CHD patients and $120 \mathrm{CHD}$ patients without statin treatment were previously enrolled in this study. All the CHD patients received statin treatment, and 63 of them were followed after 3 months of regular statin treatment. HDL sample of each patient was isolated by density gradient ultracentrifugation from fasting venous plasma, and HDL-apoCIII of each patient was measured by ELISA method.
\end{abstract}

Results: HDL-apoCIII was significantly higher in CHD patients than non-CHD patients $(p<0.05)$, and it was still an independent predictor of CHD after adjusting for other factors. Total plasma apoCIII, especially HDL-apoCIII was significantly elevated after statin treatment in CHD patients, whereas total cholesterol (TC), low density lipoprotein cholesterol (LDL-C) and apolipoprotein B (apoB) were decreased significantly $(p<0.05)$. Compared with CHD patients without diabetes mellitus (DM), the effect of statin treatment on apoCIII markers was minor in CHD patients with DM. And HDL-apoCIII correlated with plasma TG significantly in non-CHD and CHD patients $(p<0.05)$, but the correlation in CHD patients did not exist after statin treatment $(p>0.05)$.

Conclusions: HDL-apoCIII has a significant and positive association with CHD. Although conventional atherogenic lipid markers have a significantly decrease in CHD patients after statin treatment, $\mathrm{HDL}$-apoCIII has a further elevation at the same time.

Keywords: Coronary heart disease (CHD), Apolipoprotein CIII (apoCIII), Statin, High density lipoprotein (HDL)

\section{Introduction}

Coronary heart disease (CHD) is a chronic inflammatory disease characterized with dyslipidemia [1-3]. Conventional lipid markers such as total cholesterol (TC), triglyceride (TG), low-density lipoprotein cholesterol (LDL-c) and high-density lipoprotein cholesterol (HDL-c) are considered to associate with CHD positively or

\footnotetext{
* Correspondence: Ichyl_fuwai@sina.com

'The Key Laboratory of Clinical Trial Research of Cardiovascular Drugs, Ministry of Health, State Key Laboratory of Cardiovascular Diseases, Fuwai Hospital, National Center for Cardiovascular Diseases, Chinese Academy of Medical Sciences and Peking Union Medical College, Beijing, China Full list of author information is available at the end of the article
}

inversely $[4,5]$. However, not all the CHD patients have dyslipidemia, and large clinical trials also fail to find more heart benefits by further elevating HDL-c [6, 7]. Increasing evidences suggest that apolipoproteins in lipoproteins or plasma may predict CHD better $[5,8]$, and the biological function of HDL is associated with its components much more [9].

As a major component of triglyceride-rich lipoproteins (TRL), apolipoprotein CIII (apoCIII) is considered to participate in the process of CHD by disturbing lipids lipolysis and promoting inflammation response [10-12]. Studies found that apoCIII or apoCIII-containing 
lipoproteins had significant associations with CHD $[8,13,14]$. Even the antiatherogenic function of HDL was also impaired by apoCIII in CHD patients [15], and elevated apoCIII content in HDL (HDL-apoCIII) predicted the occurrence and progression of $\mathrm{CHD}$ in prospective or retrospective studies [16-18]. However, the relationship between HDL-apoCIII and CHD is still inconclusive because of the general usage of lipid-lowering drugs in almost all studies above. Lipid-lowering drugs, particularly statins were demonstrated to reduce the progression of CHD and cardiovascular event significantly by decreasing atherogenic lipids [19, 20]. To date, it is still unclear if statin treatment has the same effect on HDL-apoCIII in CHD patients. The objectives of our study are to establish the association between HDL-apoCIII and CHD, and investigate the effect of statin treatment on HDL-apoCIII in CHD patients.

\section{Method}

\section{Study design and population}

The study was approved by the Ethics Committee of the Fuwai Hospital (Approval No. 2012-382) and was registered at http://clinicaltrials.gov/ (Identification Number: NCT 01543308). A written informed consent was provided to all study patients, and the study protocol conforms to the guidelines of Declaration of Helsinki.

We conducted a hospital-based observational study. Patients without previously lipid-lowering treatment were consecutively recruited from April 2012 to March 2013 in Ward IV of Fuwai Hospital. Total 200 patients, including 80 non-CHD patients and 120 CHD patients, were entered into the study. CHD was diagnosed according to the angiographic results: stenosis $\geq 50 \%$ in at least one major coronary artery. All $120 \mathrm{CHD}$ patients received statin treatment (atorvastatin calcium 20 or $40 \mathrm{mg} /$ day, rosuvastatin calcium $10 \mathrm{mg} /$ day, pravastatin sodium $40 \mathrm{mg} /$ day, or simvastatin $40 \mathrm{mg} /$ day) regardless of whether a stent was implanted or not. Sixty-three CHD patients were followed after 3 months of regular statin treatment (detailed information about the statin treatment was listed in Additional files 3 and 4); 57 CHD patients refused to return for blood drawing. Considered the effect of Diabetes mellitus (DM) on lipids metabolism, the DM history of each patient was recorded. DM was diagnosed based on the National Cholesterol Education Program Adults Treatment Panel III or personal DM history. A total of $10 \mathrm{CHD}$ patients with DM were followed in this study, including 5 patients used acarbose ( 2 of them also used glyburide or glipizide), 2 patients used insulin, and 3 patients used metformin, Chinese medicine and dietetic treatment respectively. The exclusion criteria included patients with any kinds of tumors and infections, severe liver or kidney diseases, uncontrolled hypertension, and severe heart failure.

\section{Plasma preparation}

Venous blood was collected from each patient after overnight fasting. Fresh plasma was obtained by centrifuging at $3000 \mathrm{r} / \mathrm{min}$ at $4{ }^{\circ} \mathrm{C}$ for $10 \mathrm{~min}$ immediately, and then aliquoting the plasma for lipids measurement or storage at $-80{ }^{\circ} \mathrm{C}$ for $\mathrm{HDL}$ isolation and apoCIII measurement.

\section{HDL isolation}

As described previously [21], a HDL $(\mathrm{d}=1.063-1.240 \mathrm{~g} / \mathrm{ml})$ sample of each patient was isolated from plasma by a sequential ultracentrifugation method. Briefly, plasma was adjusted to $1.240 \mathrm{~g} / \mathrm{ml}$ by potassium bromide powder according to the Radding-Steining formula. The adjusted plasma samples were laid on the bottom of a centrifuge tube through $\mathrm{KBr}$ solution $(\mathrm{d}=1.063 \mathrm{~g} / \mathrm{ml})$ and was centrifuged by $80 \mathrm{Ti}$ Rotor ultracentrifuge (Beckman Coulter, USA) at the condition of $65000 \mathrm{r} / \mathrm{min}$ at $10{ }^{\circ} \mathrm{C}$ for $5 \mathrm{~h}$. Finally the HDL sample was collected, desalted, freeze-dried, and resolved by deionized water for measurement.

\section{Lipids measurement}

Lipid markers including TC, TG, HDL-c, LDL-c, apolipoprotein AI (ApoAI), apolipoprotein B (ApoB) and high sensitivity $\mathrm{C}$-reactive protein ( $\mathrm{HsCRP}$ ) were measured by a biochemistry analyzer in the clinical laboratory centre of Fuwai hospital, as described previously [21]. And the plasma apoCIII concentrations and HDL-apoCIII were measured by using an ELISA kit (Abcam, UK). The concentrations of HDL samples were quantified by using BCA protein assay (Applygen Technologies Inc, China). The effect of the differences of HDL sample concentrations was considered, and all the ELISA results $(\mathrm{ng} / \mathrm{ml})$ of HDL-apoCIII were adjusted by the concentrations (ug/ml) of HDL samples, and the final HDL-apoCIII unit was reported in ug/mgHDL.

\section{Statistical analysis}

All the data were expressed as mean \pm standard deviation (SD) or percent (\%). Normality was checked before any analysis. The differences between groups were analyzed by student $t$-test, paired $t$-test or Mann-Whitney $U$ test, or Wilcoxon rank test, as appropriate. A multivariate logistic regression model was used to analyze the odds ratios and $95 \%$ confidence interval of lipid markers for predicting CHD. The correlations between lipid markers were analyzed by Pearson or Spearman correlation. The statistical analysis was performed using SPSS version 17.0 (SPSS Inc., Chicago, Illinois). A $p$-value of $<0.05$ was considered to be statistically significant. 


\section{Results}

As shown Table 1, the elder males had a higher ratio of CHD $(p<0.05)$, and the results consist to epidemiologic features. Data stratified by sex were listed in Additional file 1. Totally, compared with non-CHD patients, CHD patients had significantly higher apoB and HsCRP, and significantly lower HDL-c and apoAI than non-CHD patients $(p<0.05)$. There were no significant differences of TC, TG and LDL-c between CHD and non-CHD groups in this study $(p>0.05)$. However, there was no significant difference of total plasma apoCIII between two groups $(p>0.05)$, but HDL-apoCIII was significantly higher in CHD patients than non-CHD patients $(p<0.01)$.

In order to identify the relationship between HDLapoCIII and CHD, we analyzed the associations between various lipid markers and CHD by multivariate logistic regression analysis (Table 2). HDL-apoCIII was still an independent predictor of $\mathrm{CHD}$ after adjusting for other facors $(p<0.05)$. Besides, sex, age, HDL-c, apoB and HsCRP were also significant predictors of $\mathrm{CHD}(p<0.05)$. According to the data, HDL-c has a negative, and HDLapoCIII has a positive association with CHD.

Sixty-three patients were followed in the study after 3 months of regular statin treatment, and the baseline characteristics of them were consistent with the CHD patients without follow-up data (Additional file 2, $p>0.05$ ). The plasma apoCIII, especially HDL-apoCIII, had a significant elevation after statin treatment $(p<0.05)$, whereas

Table 1 Baseline characteristics of all the patients

\begin{tabular}{lllr}
\hline Variables & $\begin{array}{l}\text { Non-CHD } \\
(n=80)\end{array}$ & $\begin{array}{l}\text { CHD } \\
(n=120)\end{array}$ & \\
\hline Male (\%) & 55.0 & 76.7 & 0.002 \\
Age (years) & $51.34 \pm 8.35$ & $55.52 \pm 10.04$ & 0.002 \\
BMl (kg/m $\left.{ }^{2}\right)$ & $26.19 \pm 3.23$ & $26.31 \pm 3.50$ & 0.697 \\
Hypertension (\%) & 47.5 & 58.3 & 0.191 \\
Diabetes (\%) & 11.3 & 19.2 & 0.247 \\
TC (mmol/L) & $4.71 \pm 0.80$ & $4.49 \pm 0.94$ & 0.088 \\
TG (mmol/L) & $1.62 \pm 1.00$ & $1.76 \pm 0.84$ & 0.079 \\
HDL-c (mmol/L) & $1.24 \pm 0.36$ & $1.02 \pm 0.23$ & $<0.001$ \\
LDL-c (mmol/L) & $3.03 \pm 0.78$ & $2.88 \pm 0.82$ & 0.199 \\
ApoAl (mmol/L) & $1.48 \pm 0.29$ & $1.34 \pm 0.24$ & 0.001 \\
ApoB (mmol/L) & $1.07 \pm 0.22$ & $1.16 \pm 0.31$ & 0.020 \\
Glucose(mmol/L) & $5.64 \pm 1.36$ & $5.43 \pm 1.56$ & 0.327 \\
HsCRP (mg/L) & $1.75 \pm 1.72$ & $3.66 \pm 3.58$ & $<0.001$ \\
ApoCIII (mg/L) & $12.68 \pm 3.74$ & $11.97 \pm 4.94$ & 0.275 \\
HDL-apoCIII (ug/mgHDL) & $21.00 \pm 11.33$ & $25.05 \pm 12.98$ & 0.024 \\
\hline
\end{tabular}

Data are expressed as mean \pm standard deviation or percent $(\%)$. $\mathrm{CHD}=$ coronary heart disease; $\mathrm{BMI}=$ body mass index $; \mathrm{TC}=$ total cholesterol; $\mathrm{TG}=$ triglyceride; $\mathrm{HDL}-\mathrm{c}=$ high density lipoprotein cholesterol; $\mathrm{LDL}-\mathrm{c}=$ low density lipoprotein cholesterol; $\mathrm{Apo}=$ apolipoprotein; $\mathrm{HsCRP}$ = high sensitivity C-reactive protein; $\mathrm{HDL}$-apoCIII = apoCIII content in HDL
Table 2 Odds ratios (OR) and $95 \%$ confidence intervals (Cl) of variables for predicting $\mathrm{CHD}$

\begin{tabular}{llll}
\hline Variables & OR & $95 \% \mathrm{Cl}$ & $p$ \\
\hline Sex & 3.02 & $1.20-7.59$ & 0.019 \\
Age & 1.07 & $1.02-1.12$ & 0.003 \\
TC & 1.03 & $0.33-3.22$ & 0.961 \\
TG & 0.83 & $0.45-1.52$ & 0.543 \\
HDL-C & 0.06 & $0.01-0.95$ & 0.046 \\
LDL-c & 0.44 & $0.13-1.50$ & 0.191 \\
ApoAl & 2.21 & $0.19-25.49$ & 0.526 \\
ApoB & 9.48 & $1.01-88.75$ & 0.049 \\
ApoCIII & 0.96 & $0.87-1.05$ & 0.345 \\
HDL-apoCIII & 1.04 & $1.00-1.08$ & 0.039 \\
HsCRP & 1.22 & $1.02-1.45$ & 0.028 \\
\hline
\end{tabular}

$\mathrm{CHD}=$ coronary heart disease; $\mathrm{TC}=$ total cholesterol; $\mathrm{TG}=$ triglyceride; $\mathrm{HDL}-\mathrm{c}=$ high density lipoprotein cholesterol; $L D L-c=$ low density lipoprotein cholesterol; Apo = apolipoprotein; HDL-apoCIII = apoCIII content in HDL

The following variables were entered in the multivariable regression model: sex, age, BMI, TC, TG, HDL-c, LDL-c, lipoprotein a, apoAl, apoB, HsCRP, apoCIII, $\mathrm{HDL}$-apoCIII and concomitant disease hypertension and diabetes mellitus

HDL-c and ApoAI were elevated, and TC, LDL-c and apoB were decreased significantly (Table $3, p<0.001$ ). Although a similar effect on lipids changes was found in different statins, rosuvastatin showed a much stronger effect on apoCIII markers than the others (Additional file 3). We did not find an obvious dose-dependent effect in different doses of atorvastain treatment (Additional file 4). As an inflammation factor, $\mathrm{HsCRP}(3.25 \pm 3.38 \mathrm{mg} / \mathrm{L}$ vs $2.22 \pm 2.49 \mathrm{mg} / \mathrm{L}$ ) was also significantly decreased after statin treatment $(p<0.01)$. In further analysis, the effect of statin treatment on apoCIII markers were minor in CHD patients with DM $(p>0.05)$, but the effect on plasma apoCIII $(11.14 \pm 3.72 \mathrm{mg} / \mathrm{L}$ vs $12.95 \pm 5.75 \mathrm{mg} / \mathrm{L})$ and HDL-apoCIII $(25.19 \pm 15.39 \mathrm{ug} / \mathrm{mgHDL}$ vs $30.84 \pm 16.92$ $\mathrm{ug} / \mathrm{mgHDL}$ ) were significant in CHD patients without DM (Additional file 5, $p<0.05$ ).

Table 3 Effect of statin treatment on lipid variables in CHD patients

\begin{tabular}{lccr}
\hline Variables & \multicolumn{2}{c}{ Statin treatment $(\mathrm{n}=63)$} & $p$ \\
\cline { 2 - 3 } & Pre-treatment & Post-treatment & \\
\hline TC $(\mathrm{mmol} / \mathrm{L})$ & $4.45 \pm 0.98$ & $3.85 \pm 0.87$ & $<0.001$ \\
TG $(\mathrm{mmol} / \mathrm{L})$ & $1.68 \pm 0.77$ & $1.54 \pm 0.76$ & 0.138 \\
HDL-c $(\mathrm{mmol} / \mathrm{L})$ & $1.05 \pm 0.25$ & $1.21 \pm 0.54$ & $<0.001$ \\
$\mathrm{LDL}-\mathrm{c}(\mathrm{mmol} / \mathrm{L})$ & $2.84 \pm 0.84$ & $2.19 \pm 0.69$ & $<0.001$ \\
ApoAl(mmol/L) & $1.37 \pm 0.24$ & $1.49 \pm 0.30$ & $<0.001$ \\
ApoB $(\mathrm{mmol} / \mathrm{L})$ & $1.16 \pm 0.34$ & $0.89 \pm 0.29$ & $<0.001$ \\
AppoCIII $(\mathrm{mg} / \mathrm{L})$ & $11.30 \pm 4.10$ & $12.93 \pm 5.71$ & 0.045 \\
HDL-apoCIII $(\mathrm{ug} / \mathrm{mgHDL})$ & $24.26 \pm 14.80$ & $29.35 \pm 16.46$ & 0.003 \\
\hline
\end{tabular}

$\mathrm{CHD}=$ coronary heart disease; $\mathrm{TC}=$ total cholesterol; $\mathrm{TG}=$ triglyceride; $\mathrm{HDL}-\mathrm{c}=$ high density lipoprotein cholesterol; LDL-C = low density lipoprotein; $A$ po = apolipoprotein; HDL-apoCIII = apoCIII content in HDL 
HDL-apoCIII had significant correlations with plasma TG in non-CHD patients and CHD patients (Fig. 1a and b, $p<0.05)$, but this correlation disappeared after statin treatment (Fig. 1c, $p>0.05$ ). However, plasma apoCIII had a moderate correlation with TG in non-CHD, CHD
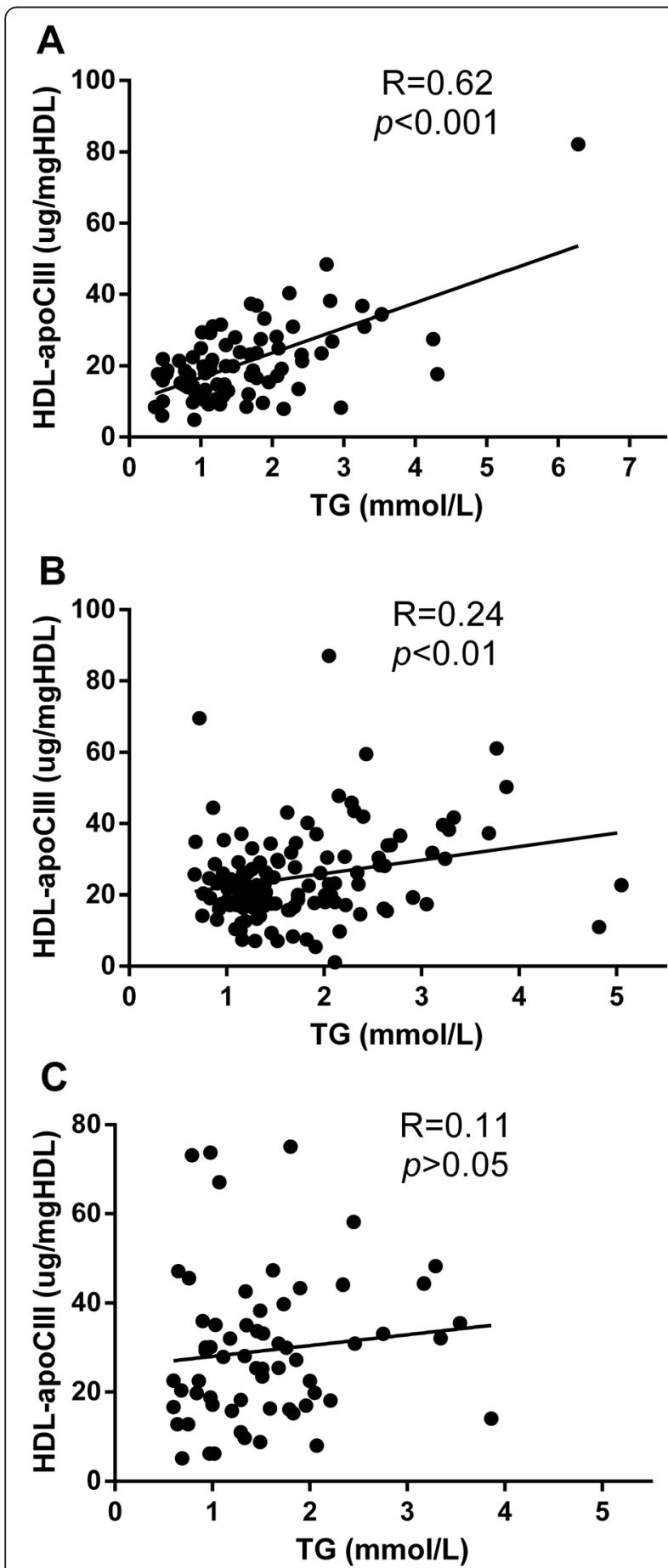

Fig. 1 Correlations between $\mathrm{HDL}$-apoCIII and plasma triglyceride in non-CHD patients (a) and CHD patients before (b) and after (c) statin treatment. $\mathrm{HDL}$-apoCIII = apoCIII content in $\mathrm{HDL}$; TG = triglyceride and statin treated CHD patients $(\mathrm{r}=0.50,0.59$ and 0.58 respectively, $p<0.001$ ); and HDL-apoCIII significantly correlated to plasma apoCIII regardless of whether statin drugs were used or not $(\mathrm{r}=0.35,0.31$ and 0.35 respectively, $p<0.05)$. In this study, we did not find significant correlations between HDL-apoCIII or plasma apoCIII and HDL-c or LDL-c $(p>0.05)$.

\section{Discussion}

In this study, we found that CHD patients had a significant higher level of HDL-apoCIII than non-CHD patients, and the association between HDL-apoCIII and CHD was still significant after adjusting for other variable factors. However, although conventional atherogenic lipid and inflammation factors were significantly decreased, there was a further elevation of HDL-apoCIII in CHD patients after statin treatment.

As an atherogenic factor, apoCIII exists in both HDL and apoB-lipoproteins, and transfers between them during the process of lipolysis [22-24]. Studies have found that apoCIII may participate in the initiation and progression of CHD through disturbing lipids metabolism, promoting the adhesion of monocyte to endothelial cell, and impairing the anti-apoptotic function of HDL $[10-12,15]$. There were also some clinical studies found that HDL-apoCIII was significantly higher in CHD patients than in non-CHD patients, which can be used to predict the occurrence and progression of CHD, as well as the recurrent cardiovascular event $[8,16,17]$. In this study, in order to avoid potential effects of the concentration of HDL samples, we adjusted HDL-apoCIII values by the concentration of $\mathrm{HDL}$ after measuring apoCIII of HDL samples. And we also found a significantly higher HDL-apoCIII in CHD patients than nonCHD patients, and it still associated with CHD significantly after adjusting for other variable factors. To some extent, HDL-apoCIII was a better predictor of CHD than most conventional lipid markers.

This is the first time we quantitatively measured a significant elevation of HDL-apoCIII in CHD patients with adjustment of the effect of HDL sample, and established the association between HDL-apoCIII and CHD without the effect of lipid-lowering drugs. We also found an inverse association of HDL-c and HDL-apoCIII with CHD, a higher HDL-c predicts a lower risk of $\mathrm{CHD}$, but a higher HDL-apoCIII predicts a higher risk of $\mathrm{CHD}$, this finding was supported by the results of previous studies $[4,5,17,18]$. Studies have found that HDL lost its antiinflammatory and anti-atherogenic function in end-stage renal diseases or acute phase response [25-27]. Perhaps this condition also existed in CHD patients, and the elevated HDL-apoCIII may be partly responsible for the dysfunction of HDL in CHD patients, and the occurrence and progression of $\mathrm{CHD}$. However, considered the 
inhibitory effect of apoCIII on lipolysis [24], the elevated HDL-apoCIII may delay the further lipolysis of HDL in CHD patients also. So, the biological function of HDLapoCIII in CHD patients may need further more study.

Lipid-lowering drugs, especially statins, are famous for their cardiovascular protective role by decreasing atherogenic, elevating antiatherogenic lipids and inhibiting inflammation response [19, 20, 28, 29]. An earlier study indicated that lipid-lowering drugs (colestipol hydrochloride and niacin) significantly decreased conventional lipids and delayed the progression of CHD, but they significantly increased HDL-apoCIII [18]. Another study also found although most CHD patients received statin treatment, they still had higher apoCIII contents in plasma and HDL, and the elevated apoCIII in HDL was responsible for the impaired antiapoptotic effect of HDL [15]. There was little studies reported that atorvastatin can decrease plasma apoCIII [30], but this result was still controversial [31]. In this study, we found a similar effect of statin treatment on atherogenic lipids and inflammation factor as previous studies $[19,20$, 29, 32], however, not as expected, plasma apoCIII, especially HDL-apoCIII has a significant elevation at the same time. The result was partly consistent to previous studies [15, 18], and all the results favored the theory of apoCIII transfer, increased lipolysis of TRL leads to increased transfer of apoCIII to HDL. However, no matter the elevation of HDL-apoCIII was naturally or caused by statin treatment, an excessive elevated HDL-apoCIII may impair, and even counteract the antiatherogenic effect of HDL and/or the benefits of statin treatment. All the results might explain why further elevating HDL-c could not increase heart benefits $[6,7]$, and why there were still residual cardiovascular risks after statin treatment in CHD patients [33, 34].

We found a similar effect of statin treatment on lipid markers in CHD patients with DM or not. Perhaps because of the small case number or the interaction of antidiabetic drugs, we did not find a significant effect of statin treatment on apoCIII markers in patients with DM $(n=10)$. Our results were inconsistent with a previous study, which found a decrease of apoCIII in plasma and HDL in DM patients after atorvastatin treatment [35]. DM patients have higher risk of CHD than nondiabetic patients [36, 37], although statin treatment reduces the risk of CHD by decreasing lipids, it also leads to an increased risk of DM [38]. To date, there is little information about the differences of effect of statins on lipid markers among CHD patients, DM patients and CHD patients with DM, maybe the effect are different among them.

As reported previously [39], we also found a significant correlation between HDL-apoCIII and TG. Perhaps because of the effect of dyslipidemia and statin drugs, the correlation was weaker in CHD patients, and disappeared after statin treatment. However, the correlation between apoCIII and TG or HDL-apoCIII was not affected by statin drugs. As a part of the theory of apoCIII transfer, we did not find a significant correlation between HDL-apoCIII and HDL-c or LDL-c.

\section{Limitations}

An important limitation of our study was that there were no placebo controls because all the CHD patients received statin treatmen. However, to some extent, the self-control method and the consistency of the results to previous studies favor the accuracy of the study. Although ultracentrifuge is a golden standard to isolate HDL, and we adjusted potential effect of HDL samples on the apoCIII results as far as possible, there may be some bias existing inevitably during the procedure.

\section{Conclusions}

Our study found that, HDL-apoCIII has a positive and significant association with CHD. Although statin treatment for $\mathrm{CHD}$ patients could decrease conventional atherogenic lipid markers significantly, HDL-apoCIII also has a significant elevation. The results may indicate a new aspect for studying CHD and evaluating the effect of statin treatment on the biological function of HDL in CHD patients.

\section{Additional files}

Additional file 1: Baseline characteristics of all patients stratified by sex. (DOC $39 \mathrm{~kb}$ )

Additional file 2: Baseline characteristics of CHD patients with followed data or not. (DOC $37 \mathrm{~kb}$ )

Additional file 3: Effect of different types of statins on lipid variables in CHD patients. (DOC $36 \mathrm{~kb}$ )

Additional file 4: Effect of different doses of atorvastatin on lipid variables in CHD patients. (DOC $36 \mathrm{~kb}$ )

Additional file 5: Effect of statin treatment on lipid variables in CHD patients with DM or not. (DOC $37 \mathrm{~kb}$ )

\section{Abbreviations}

CHD: Coronary heart disease; DM: Diabetes mellitus; TC: Total cholesterol; TG: Triglyceride; HDL-c: High density lipoprotein cholesterol; LDL-c: Low density lipoprotein cholesterol; Apo: Apolipoprotein; HsCRP: High sensitivity C-reactive protein; HDL-apoCIII: apoCIII content in HDL; TRL: Triglyceride-rich lipoproteins.

\section{Competing interests}

The authors declare that they have no competing interests.

\section{Authors' contributions}

$\mathrm{XX}-\mathrm{W}$ and $\mathrm{LH}$ took part in study design, data analysis and writing the manuscript WD-X, HL and ZH recruited the patients, isolated high density protein cholesterol samples and assayed the data. LY-S initiated and designed the study. All authors read and approved the final version of this manuscript. 


\section{Acknowledgements}

This work was financially supported by the Key Laboratory Construction Project from the Ministry of Health and the National Science and Technology Major Project (No. 2011ZX09304) of the Ministry of Science and Technology of the People's Republic of China.

\section{Author details}

${ }^{1}$ The Key Laboratory of Clinical Trial Research of Cardiovascular Drugs, Ministry of Health, State Key Laboratory of Cardiovascular Diseases, Fuwai Hospital, National Center for Cardiovascular Diseases, Chinese Academy of Medical Sciences and Peking Union Medical College, Beijing, China. ²Department of Cardiology, Wuxi People' Hospital Affiliated to Nanjing Medical University, Wuxi, Jiangsu, China.

\section{Received: 28 August 2015 Accepted: 3 October 2015} Published online: 09 October 2015

\section{References}

1. Hansson GK. Inflammation, atherosclerosis, and coronary artery disease N Engl J Med. 2005;352(16):1685-95.

2. The Expert Panel. Third Report of the National Cholesterol Education Program (NCEP) Expert Panel on Detection, Evaluation, and Treatment of High Blood Cholesterol in Adults (Adult Treatment Panel III) final report. Circulation. 2002:106:3143-421.

3. Steinberg D. Hypercholesterolemia and inflammation in atherogenesis: Two sides of the same coin. Mol Nutr Food Res. 2005:49(11):995-8.

4. Sharrett AR, Ballantyne CM, Coady SA, Heiss G, Sorle PD, Catellier D, et al. Coronary heart disease prediction from lipoprotein cholesterol levels, triglycerides, lipoprotein(a), apolipoprotiens A-I and B, and HDL density subfractions: The atherosclerosis Risk in Communities (ARIC) study. Circulation. 2001;104(10):1108-13.

5. Emerging Risk Factors Collaboration, Di Angelantonio E, Sarwar N, Perry P, Kaptoge S, Ray KK, et al. Major lipids, apolipoproteins, and risk of vascular disease. JAMA. 2009;302(18):1993-2000.

6. Nissen SE, Tardif JC, Nicholls SJ, Revkin JH, Shear CL, Duggan WT, et al. Effect of torcetrapib on the progression of coronary atherosclerosis. $\mathrm{N}$ Engl J Med. 2007;356(13):1304-16.

7. Barter PJ, Caulfield M, Eriksson M, Grundy SM, Kastelein JJ, Komajda M, et al. Effects of torcetrapib in patients at high risk for coronary events. N Engl J Med. 2007;357(21):2109-22

8. Sacks FM, Alaupovic P, Moye LA, Cole TG, Sussex B, Stampfer MJ, et al. VLDL, apolipoprotiens $\mathrm{B}, \mathrm{CIII}$, and $\mathrm{E}$, and risk of recurrent coronary events in the Cholesterol and Recurrent Events (CARE) Trial. Circulation. 2000;102(16):1886-92.

9. Vaisar T. Proteomics investigations of HDL: challenges and promise. Curr Vasc Pharmacol. 2012;10(4):410-21.

10. Zheng C, Khoo C, Furtado J, Sacks FM. Apolipoprotien C-III and the metabolic basis for hypertriglyceridemia and the dense low-density lipoprotein phenotype. Circulation. 2012;121(15):1722-34.

11. Kawakami A, Aikawa M, Alcaide P, Luscinskas FW, Libby P, Sacks FM Apolipoprotien CIII induces expression of vascular cell adhesion molecule-1 in vascular endothelial cells and increases adhesion of monocytic cells. Circulation. 2006;114(7):681-7

12. Kawakami A, Aikawa M, Libby P, Alcaide P, Luscinskas FW, Sacks FM Apolipoprotien CIII in apoliprotein B lipoproteins enhances the adhesion of human monocytic cells to endothelial cells. Circulation. 2006;113(5):691-700.

13. Mendivil CO, Rimm EB, Furtado J, Chiuve SE, Sacks FM. Low-density lipoproteins containing apolipoprotien C-III and the risk of coronary heart disease. Circulation. 2011;124(19):2065-72

14. Scheffer PG, Teerlink T, Dekker JM, Bos G, Nijpels G, Diamant M, et al. Increased plasma apolipoprotein C-III concentration independently predicts cardiovascular mortality: the Hoorn Study. Clin Chem. 2008;54(8):1325-30.

15. Riwanto M, Rohrer $L$, Roschitzki B, Besler C, Mocharla P, Mueller M, et al. Altered activation of endothelial anti- and proapoptotic pathways by high-density lipoprotein from patients with coronary disease: role of high-density lipoprotein-proteome remodeling. Circulation. 2013;127(8):891-904.

16. Jensen MK, Rimm EB, Furtado JD, Sacks FM. Apolipoprotein C-III as a potential modulator of the association between $\mathrm{HDL}$-cholesterol and incident coronary heart disease. J Am Heart Assoc. 2012;1 (2):e000232. doi:10.1161/JAHA.111.000232
17. Chang PY, Lee CM, Hsu HC, Lin HJ, Chien $\mathrm{KL}$, Chen $\mathrm{CH}$, et al. Identification of the HDL-ApoCIII to VLDL-ApoCIII ratio as a predictor of coronary artery disease in the general population: the Chin-Shan Community Cardiovascular Cohort (CCCC) study in Taiwan. Lipids Health Dis. 2012:11:162

18. Blankenhorn DH, Alaupovic P, Wickam E, Chin HP, Azen SP. Prediction of angiographic change in native human coronary arteries and aortocoronary bypass grafts. Lipid and nonlipid factors. Circulation. 1990;81(2):470-6.

19. Law MR, Wald NJ, Rudnicka AR. Quantifying effect of statins on low density lipoprotein cholesterol, ischaemic heart disease, and stroke: systematic review and meta-analysis. BMJ. 2003;326(7404):1423.

20. Jones PH, Davidson MH, Stein EA, Bays HE, McKenney JM, Miller E, et al. Comparison of the efficacy and safety of rosuvastatin, simvastatin, and pravastatin across doses (STELLAR*Trial). Am J Cardiol. 2003;92(2):152-60.

21. Yan LR, Wang DX, Liu H, Zhang XX, Zhao H, Hua L, et al. A pro-atherogenic $\mathrm{HDL}$ profile in coronary heart disease patients: an iTRAQ labeling-based proteomic approach. PLoS One. 2014;9(5):e98368.

22. Ooi EM, Barrett PH, Chan DC, Watts GF. Apolipoprotein C-III: understanding an emerging cardiovascular risk factor. Clin Sci. 2008;114(10):611-24.

23. Malmendier CL, Lontie JF, Grutman GA, Delcroix C. Metabolism of apolipoprotein C-III in normolipemic human subjects. Atherosclerosis. 1988;69(1):51-9.

24. Tornoci L, Scheraldi CA, Li X, Ide H, Goldberg IJ, Le NA. Abnormal activation of lipoprotein lipase by non-equilibrating apoC-II: further evidence for the presence of non-equilibrating pools of apolipoproteins C-II and C-III in plasma lipoproteins. J Lipid Res. 1993;34(10):1793-803.

25. Speer T, Rohrer L, Blyszczuk P, Shroff R, Kuschnerus K, Krankel N, et al. Abnormal high-density lipoprotein induces endothelial dysfunction via activation of Toll-like receptor-2. Immunity. 2013;38(4):754-68.

26. Tolle M, Huang $T$, Schuchardt M, Jankowski V, Prufer N, Jankowski J, et al High-density lipoprotein loses its anti-inflammatory capacity by accumulation of pro-inflammatory-serum amyloid A. Cardiovasc Res. 2012;94(1):154-62

27. Van Lenten BJ, Hama SY, de Bee FC, Stafforini DM, Mclntyre TM, Prescott $\mathrm{SM}$, et al. Anti-inflammatory HDL becomes pro-inflammatory during the acute phase response. Loss of protective effect of $H D L$ against LDL oxidation in aortic wall cell cocultures. J Clin Invest. 1995;96(6):2758-67.

28. Kim YS, Ahn Y, Hong MH, Kim KH, Park HW, Hong YJ, et al. Rosuvastatin suppresses the inflammatory responses through inhibition of c-Jun $\mathrm{N}$-terminal kinase and nuclear facto-kappaB in endothelial cells. J Cardiovasc Pharmacol. 2007:49(6):376-83.

29. Ridker PM, Danielson E, Fonseca FA, Genest J, Gotto Jr AM, Kastelein JJ, et al. Rosuvastatin to prevent vascular events in men and women with elevated C-reactive protein. N Engl J Med. 2008;359(21):2195-207.

30. Le NA, Innis-Whitehouse W, Li X, Bakker-Arkema R, Black D, Brown W. Lipid and apolipoprotein levels and distribution in patients with hypertriglyceridemia: effect of triglyceride reductions with atorvastatin. Metabolism. 2000;49(2):167-77.

31. Alaupovic P, Fesmire JD, Hunnighake D, Domanski M, Forman S, Knatterud $\mathrm{GL}$, et al. The effect of aggressive and moderate lowering of LDL-cholesterol and low dose anticoagulation on plasma lipids, apolipoproteins and lipoprotein families in post coronary artery bypass graft trial. Atherosclerosis. 1999;146(2):369-79.

32. Zheng C, Azcutia V, Aikawa E, Fiqueiredo JL, Croce K, Sonoki H, et al. Statins suppress apolipoprotein CIII-induced vascular endothelial cell activation and monocyte adhesion. Eur Heart J. 2013;34(8):615-24.

33. Singh IM, Shishehbor MH, Ansell BJ. High-density lipoprotein as a therapeutic target: a systematic review. JAMA. 2007;298(7):786-98.

34. Briel M, Ferreira-Gonzalez I, You JJ, Karanicolas PJ, Aki EA, Wu P, et al. Association between change in high density lipoprotein cholesterol and cardiovascular disease morbidity and mortality: systematic review and meta-regression analysis. BMJ. 2009;338:b92.

35. Dallinga-Thie GM, Berk-Planken II, Bootsma AH, Jansen H. Atorvastatin decreases apolipoprotein C-III in apolipoprotein B-containing lipoprotein and HDL in type 2 diabetes: a potential mechanism to lower plasma triglycerides. Diabetes Care. 2004;27(6):1358-64.

36. Stamler J, Vaccaro O, Neaton JD, Wentworth D. Diabetes, other risk factors, and 12-yr cardiovascular mortality for men screened in the Multiple Risk Factor Intervention Trial. Diabetes Care. 1993;16(2):434-44.

37. Lee SJ, Campos H, Moye LA, Sacks FM. LDL containing apolipoprotein CIII is an independent risk factor for coronary events in diabetic patients. Arterioscler Thromb Vasc Biol. 2003;23(5):853-8. 
38. Ridker PM, Pradhan A, MacFadyen JG, Libby P, Glynn RJ. Cardiovascular benefits and diabetes risks of statin therapy in primary prevention: an analysis from the JUPITER trial. Lancet. 2012;380(9841):565-71.

39. Onat A, Hergenc G, Sansoy V, Fobker M, Ceyhan K, Toprak S, et al. Apolipoprotein c-III, a strong discrminant of coronary risk in men and a determinant of the metabolic syndrome in both genders.

Atherosclerosis. 2003;168(1):81-9.

Submit your next manuscript to BioMed Central and take full advantage of:

- Convenient online submission

- Thorough peer review

- No space constraints or color figure charges

- Immediate publication on acceptance

- Inclusion in PubMed, CAS, Scopus and Google Scholar

- Research which is freely available for redistribution 